\title{
Multiplicity results for the Kirchhoff type equation via critical groups
}

\author{
Zhenting Wang ${ }^{1}$, Mingzheng Sun ${ }^{1 *} \mathbb{D}$, Yutong Chen ${ }^{2}$ and Leiga Zhao ${ }^{3}$
}

\footnotetext{
$\overline{\text { "Correspondence: suncut@163.com }}$ ${ }^{1}$ College of Sciences, North China University of Technology, Beijing, China

Full list of author information is available at the end of the article
}

\begin{abstract}
In this paper, we will compute critical groups at zero for the Kirchhoff type equation using the property that critical groups are invariant under homotopies preserving isolatedness of critical points. Using this results, we can get more nontrivial solutions when the functional of this equation is coercive.
\end{abstract}

MSC: $35 \mathrm{~J} 20 ; 35 \mathrm{~B} 34 ; 58 \mathrm{E} 05$

Keywords: Kirchhoff type equations; Multiple solutions; Morse theory

\section{Introduction}

Let $\Omega$ be a bounded domain of $\mathbb{R}^{N}$ with sufficiently regular boundary $\partial \Omega$, and we study the following Kirchhoff type equation:

$$
\begin{cases}-\left(a+b \int_{\Omega}|\nabla u|^{2} d x\right) \Delta u=f(x, u) & \text { in } \Omega \\ u=0 & \text { on } \partial \Omega\end{cases}
$$

where $a, b>0$ are real constants. The problem (1.1) is related to the Kirchhoff's model, we refer to $[8,10]$ for details and further references.

Moreover, if we assume that

$\left(f_{0}\right) f \in \mathcal{C}^{1}(\bar{\Omega} \times \mathbb{R}, \mathbb{R}), f(x, 0)=0$ and there is $c>0$ such that

$$
\left|f^{\prime}(x, u)\right| \leq c\left(1+|u|^{\gamma-2}\right), \quad \text { for some } 2 \leq \gamma<2^{*}= \begin{cases}+\infty, & N=1,2 \\ \frac{2 N}{N-2}, & N \geq 3\end{cases}
$$

then weak solutions of Eq. (1.1) correspond to critical points of the $\mathcal{C}^{2}$ functional $I$ : $H_{0}^{1}(\Omega) \rightarrow \mathbb{R}$ defined by

$$
I(u)=\frac{a}{2}\|u\|^{2}+\frac{b}{4}\|u\|^{4}-\int_{\Omega} F(x, u) d x
$$

where $F(x, u)=\int_{0}^{u} f(x, t) d t$, and $H_{0}^{1}(\Omega)$ is the Hilbert space endowed with the norm

$$
\|u\|=\|\nabla u\|_{2}=\left(\int_{\Omega}|\nabla u|^{2} d x\right)^{1 / 2} .
$$

(c) The Author(s) 2018. This article is distributed under the terms of the Creative Commons Attribution 4.0 International License (http://creativecommons.org/licenses/by/4.0/), which permits unrestricted use, distribution, and reproduction in any medium, provided you give appropriate credit to the original author(s) and the source, provide a link to the Creative Commons license, and indicate if changes were made. 
In the sequel, we assume

$$
0<\lambda_{1}<\lambda_{2} \leq \cdots \leq \lambda_{k} \leq \cdots
$$

are the eigenvalues of $-\Delta$ in $H_{0}^{1}(\Omega)$.

In recent years, there have been many papers which have studied the Kirchhoff type problems by variational methods. When the nonlinearity $f$ is superlinear, the existence results of solutions can be found in $[13,18,19,26]$, and for the case where the nonlinearity is asymptotically linear, we refer to $[4,15,24,26]$ for details and further references. For example, by using the condition

$$
\lim _{t \rightarrow 0} \frac{f(x, t)}{a t}=p(x)
$$

where $0 \leq p(x) \in L^{\infty}(\Omega)$ and $\|p(x)\|_{\infty}<\lambda_{1}$, one shows [4] that 0 is a local minimizer of $I$. Moreover, the authors in [19] assume

$$
\frac{a}{2} \lambda_{k} t^{2}+C_{1} t^{4} \leq F(x, t) \leq \frac{a}{2} \lambda_{k+1} t^{2}+C_{2} t^{4}, \quad \text { for }|t|<\delta,
$$

where $\delta, C_{1}, C_{2}$ are positive constants, and they show that the functional $I$ has a local linking at zero.

In particular, using the Yang index, the authors in [15] consider the eigenvalue problem

$$
\begin{cases}-\|u\|^{2} \Delta u=\mu u^{3} & \text { in } \Omega, \\ u=0 & \text { on } \partial \Omega,\end{cases}
$$

and get an unbounded sequence of eigenvalues

$$
0<\mu_{1}<\mu_{2} \leq \cdots \leq \mu_{k} \leq \cdots
$$

Furthermore, using this sequence of eigenvalues, when the nonlinearity $f$ is superlinear near zero but asymptotically 4 -linear at infinity, in [18] one computes the relevant critical groups and obtains nontrivial solutions.

In this paper, the main aim is to give some results on the critical groups estimates at zero for $I$ and its applications to the existence and multiplicity results for equation (1.1) by Morse theory. Therefore, we recall the following notions (see [2, 14]). We assume that $u_{0}$ is an isolated critical point of $I, U$ is an isolated neighborhood of $u_{0}$, and $I\left(u_{0}\right)=c \in \mathbb{R}$, the group

$$
C_{*}\left(I, u_{0}\right)=H_{*}\left(I^{c} \cap U, I^{c} \cap U \backslash\left\{u_{0}\right\}\right), \quad * \in \mathbb{N}_{0}=\{0,1,2, \ldots\},
$$

is called the $*$ th critical group of the functional $I$ at $u_{0}$, where $I^{c}=\left\{u \in H_{0}^{1}(\Omega): I(u) \leq c\right\}$, and $H_{*}(\cdot, \cdot)$ are the singular relative homological groups with a coefficient group $\mathbb{F}$.

We impose on $f$ the following non-resonance and resonance conditions:

$\left(f_{1}\right)$ there exists $\lambda \in \mathbb{R}$ such that

$$
\lim _{|u| \rightarrow 0} \frac{f(x, u)}{a u}=\lambda, \quad \text { uniformly in } x \in \Omega
$$


$\left(f_{2}\right)$ there exists $\alpha>0$ such that

$$
\begin{aligned}
u g(x, u) & \leq 0, \quad \text { for }|u| \leq \alpha, x \in \Omega, \\
\text { where } g(x, u) & =f(x, u)-a \lambda_{1} u .
\end{aligned}
$$

Our results read as follows.

Theorem 1.1 Assume that $\left(f_{0}\right)$ and $\left(f_{1}\right)$ hold. If $\lambda \in\left(\lambda_{k}, \lambda_{k+1}\right)$, then $u=0$ is an isolated critical point of I such that

$$
C_{*}(I, 0)=\delta_{*, k} \mathbb{F} \text {. }
$$

Theorem 1.2 Assume that $\left(f_{0}\right),\left(f_{1}\right)$ and $\left(f_{2}\right)$ hold. If $\lambda=\lambda_{1}$, then $u=0$ is an isolated critical point of I such that

$$
C_{*}(I, 0)=\delta_{*, 0} \mathbb{F} .
$$

Remark 1 Note that, for the semilinear elliptic equation, i.e., $b=0$, Theorem 1.1 can be found in [2], now we can generalize the same results to Eq. (1.1) with any $b>0$. However, we cannot directly use the methods in [2], because there are many difficulties to get the critical group estimates for the functional $I$. For example, although we can get a space decomposition according to the eigenfunctions which is the basis of linking theorem by $\left(f_{1}\right)$, the second derivative of $I$ in each critical point is complex, so that we are not sure that the generalized Morse splitting lemma can be used. In spite of these difficulties, we can obtain critical groups estimates at zero by using the basic properties of critical groups (see [3]), that is, critical groups are invariant under homotopies preserving isolatedness of critical points.

Remark 2 Obviously, $\left(f_{2}\right)$ is known as one of the sign conditions in resonance problems. For the results of sign conditions with $b=0$ we refer to $[9,11,16,17]$ for details and further references.

Using Theorem 1.1, we can also obtain some multiplicity results for Eq. (1.1). We make the following assumption:

$\left(f_{3}\right)$ there exist $M>0$ and $\beta<\frac{a \lambda_{1}}{2}$ such that

$$
F(x, u)-\frac{b}{4} \mu_{1}|u|^{4} \leq \beta u^{2}, \quad \text { for }|u| \geq M, x \in \Omega,
$$

and our next result reads as follows.

Theorem 1.3 Assume that $N \leq 3,\left(f_{0}\right),\left(f_{1}\right)$ and $\left(f_{3}\right)$ hold. If $\lambda \in\left(\lambda_{k}, \lambda_{k+1}\right)$ with $k \geq 2$, then Eq. (1.1) has at least three nontrivial solutions.

Remark 3 Using similar conditions, the paper [25] has studied the Kirchhoff type equations involving the nonlocal fractional $p$-Laplacian and can get at least two nontrivial solutions by the three-critical point theorem (see [12, Theorem 2.1]). Because of the exact calculations of the critical groups at zero, our theorem can get more nontrivial solutions. Then our result is new. 
Remark 4 For the semilinear elliptic equation, if the condition

$$
\lim _{|u| \rightarrow \infty}\left(F(x, u)-\frac{1}{2} \lambda_{1}|u|^{2}\right) d x=-\infty, \quad x \in \Omega,
$$

holds, then the functional $I$ with $b=0$ is coercive ([7]). For Eq. (1.1), because of the existence of Laplacian operator, we can also prove that the functional $I$ with $b>0$ is coercive with $\left(f_{3}\right)$. For other results of $(1.1)$ we refer to $[5,7,20-23,25]$ and references therein.

This paper is organized as follows. The proofs of Theorems 1.1-1.3 are given in Sects. 24 , respectively. In the sequel, we use the letter $C$ to denote a suitable positive constant whose value may change from line to line.

\section{Proof of Theorem 1.1}

We first recall some notions and results for Morse theory (see, e.g., [2]). By $\left(f_{1}\right)$, the functional $I$ is a $C^{2}$ functional with Fréchet derivatives

$$
\left\langle I^{\prime}(u), w\right\rangle=a \int_{\Omega} \nabla u \nabla w d x+b\|u\|^{2} \int_{\Omega} \nabla u \nabla w d x-\int_{\Omega} f(x, u) w d x,
$$

for all $u, w \in H_{0}^{1}(\Omega)$.

Definition 2.1 If every sequence $\left\{u_{n}\right\} \subset H_{0}^{1}(\Omega)$ with

$$
I\left(u_{n}\right) \text { being bounded, } \quad I^{\prime}\left(u_{n}\right) \rightarrow 0, \quad \text { as } n \rightarrow \infty,
$$

possesses a convergent subsequence, then the functional $I$ is said to satisfy the PalaisSmale (for short (P.S)) condition.

Proposition $2.2([3])$ Assume $\tau \in[0,1]$, let $\Phi_{\tau} \in C^{1}\left(H_{0}^{1}(\Omega)\right)$ and

$$
u_{0} \in K\left(\Phi_{\tau}\right)=\left\{u \in H_{0}^{1}(\Omega): \Phi_{\tau}^{\prime}(u)=0\right\} .
$$

If $U \subset H_{0}^{1}(\Omega)$ is a closed neighborhood of $u_{0}$ such that

(i) $\Phi_{\tau}$ satisfies the $(P . S)$ condition in $U$ for all $\tau \in[0,1]$,

(ii) $K\left(\Phi_{\tau}\right) \cap U=\left\{u_{0}\right\}$ for all $\tau \in[0,1]$,

(iii) the mapping $\tau \rightarrow \Phi_{\tau}$ is continuous between $[0,1]$ and $C^{1}(U)$, then we have

$$
C_{*}\left(\Phi_{0}, u_{0}\right)=C_{*}\left(\Phi_{1}, u_{0}\right), \quad * \in \mathbb{N}_{0} .
$$

Lemma 2.3 Assume that $\left(f_{0}\right)$ and $\left(f_{1}\right)$ hold. If $\lambda \in\left(\lambda_{k}, \lambda_{k+1}\right)$ then $u=0$ is an isolated critical point of $I$.

Proof Clearly, by $f(x, 0)=0$ we have $u=0$ is a critical point of $I$. To see that $u=0$ is isolated, we argue by contradiction: assume that there exists a sequence $\left(u_{n}\right)$ in $H_{0}^{1}(\Omega) \backslash\{0\}$ such that

$$
\begin{cases}u_{n} \rightarrow 0, & \text { in } H_{0}^{1}(\Omega) \\ I^{\prime}\left(u_{n}\right)=0, & \forall n \geq 1\end{cases}
$$


Now, we set for all $n \geq 1, v_{n}=u_{n} /\left\|u_{n}\right\|$, and passing to a further subsequence we can assume that

$$
\begin{cases}v_{n} \rightarrow v, & \text { weakly in } H_{0}^{1}(\Omega), \\ v_{n} \rightarrow v, & \text { strongly in } L^{2}(\Omega), \\ v_{n}(x) \rightarrow v(x), & \text { a.e. } x \in \Omega .\end{cases}
$$

From (2.1), for all $n \geq 1$ and $w \in H_{0}^{1}(\Omega)$, we have

$$
\begin{aligned}
0 & =\frac{\left\langle I^{\prime}\left(u_{n}\right), w\right\rangle}{a\left\|u_{n}\right\|} \\
& =\int_{\Omega} \nabla v_{n} \nabla w d x+\frac{b}{a}\left\|u_{n}\right\|^{2} \int_{\Omega} \nabla v_{n} \nabla w d x-\int_{\Omega} \frac{f\left(x, u_{n}\right)}{a\left\|u_{n}\right\|} w d x .
\end{aligned}
$$

By $\left(f_{1}\right)$ we have

$$
\frac{f\left(x, u_{n}\right)}{a\left\|u_{n}\right\|}=\frac{f\left(x, u_{n}\right)}{a u_{n}} \frac{u_{n}}{\left\|u_{n}\right\|} \rightarrow \lambda v \quad \text { in } L^{2}(\Omega)
$$

so the sequence $\left(\frac{f\left(x, u_{n}\right)}{a\left\|u_{n}\right\|}\right)$ is bounded in $L^{2}(\Omega)$.

Choosing $w=v_{n}-v$ in (2.3) and using the Hölder inequality we get

$$
\left(1+\frac{b}{a}\left\|u_{n}\right\|^{2}\right) \int_{\Omega} \nabla v_{n} \nabla\left(v_{n}-v\right) d x \leq\left\|\frac{f\left(x, u_{n}\right)}{a\left\|u_{n}\right\|}\right\|_{2}\left\|v_{n}-v\right\|_{2}
$$

and the latter tends to 0 as $n \rightarrow \infty$. Therefore we have $v_{n} \rightarrow v$ in $H_{0}^{1}(\Omega)$ and in particular $\|v\|=1$.

Since $\left\|u_{n}\right\| \rightarrow 0(n \rightarrow \infty)$ and (2.2) holds, when we pass to the limit in (2.3) again and using (2.4), we get

$$
\int_{\Omega} \nabla v \nabla w d x=\lambda \int_{\Omega} v w d x
$$

which implies that $\lambda$ is an eigenvalue of $-\Delta$ with $v$ as an associated eigenfunction, contrary to the assumption $\lambda \in\left(\lambda_{k}, \lambda_{k+1}\right)$. The proof is completed.

Proof of Theorem 1.1 From Lemma 2.3, we know that $u=0$ is an isolated critical point of $I$. Next, we will use Proposition 2.2 to compute the critical groups of zero.

(1) First we define the $C^{2}$ functional

$$
I_{a}(u)=\frac{a}{2}\|u\|^{2}-\int_{\Omega} F(x, u) d x
$$

then, by $\left(f_{1}\right)$ with $\lambda \in\left(\lambda_{k}, \lambda_{k+1}\right)$, we know that $u=0$ is an isolated nondegenerate critical point of $I_{a}$ such that (see [2])

$$
C_{*}\left(I_{a}, 0\right)=\delta_{*, k} \mathbb{F} \text {. }
$$


(2) Now define a homotopy by setting for $u \in H_{0}^{1}(\Omega)$,

$$
J_{s}(u)=s I(u)+(1-s) I_{a}(u), \quad s \in[0,1] .
$$

Then, by $\left(f_{0}\right), J_{s} \in C^{1}\left(H_{0}^{1}(\Omega), \mathbb{R}\right)$ and satisfies (P.S) condition on the bounded domain in $H_{0}^{1}(\Omega)$ for any $s \in[0,1]$. Clearly, $u=0$ is a critical point for all $s \in[0,1]$. We claim that there is a neighborhood $U$ of 0 such that $u=0$ is the only critical point of $J_{s}$ in $U$ for all $s \in[0,1]$.

By contradiction, we assume that there exist sequences $s_{n} \in[0,1]$ and $\left(u_{n}\right)$ in $H_{0}^{1}(\Omega) \backslash\{0\}$ such that

$$
\begin{cases}u_{n} \rightarrow 0, & \text { in } H_{0}^{1}(\Omega) \\ J_{s_{n}}{ }^{\prime}\left(u_{n}\right)=0, & \forall n \geq 1\end{cases}
$$

If we set $v_{n}=u_{n} /\left\|u_{n}\right\|$, then passing to a further subsequence we can assume that

$$
\begin{cases}v_{n} \rightarrow v, & \text { weakly in } H_{0}^{1}(\Omega), \\ v_{n} \rightarrow v, & \text { strongly in } L^{2}(\Omega) \\ v_{n}(x) \rightarrow v(x), & \text { a.e. } x \in \Omega .\end{cases}
$$

For any $w \in H_{0}^{1}(\Omega)$, using (2.6) and (2.7) we get

$$
\begin{aligned}
0= & \frac{\left\langle{J_{s_{n}}}^{\prime}\left(u_{n}\right), w\right\rangle}{a\left\|u_{n}\right\|} \\
= & \int_{\Omega} \nabla v_{n} \nabla w d x+\frac{s_{n} b}{a}\left\|u_{n}\right\|^{2} \int_{\Omega} \nabla v_{n} \nabla w d x \\
& -\int_{\Omega} \frac{f\left(x, u_{n}\right)}{a u_{n}} v_{n} w d x .
\end{aligned}
$$

Using the methods in the proof of Lemma 2.3, we deduce that $v_{n} \rightarrow v$ in $H_{0}^{1}(\Omega)$ and $\|v\|=1$. Passing to the limit in (2.8) again we get a contradiction.

(3) Now, by the homotopy invariance of the critical groups in Proposition 2.2, we have

$$
C_{*}\left(J_{0}, 0\right)=C_{*}\left(I_{a}, 0\right)=C_{*}(I, 0)=C_{*}\left(J_{1}, 0\right) .
$$

Then (2.5) and (2.9) give

$$
C_{*}(I, 0)=\delta_{*, k} \mathbb{F} .
$$

The proof is completed.

\section{Proof of Theorem 1.2}

Now, we give the proof of Theorem 1.2.

Lemma 3.1 Assume that $\left(f_{0}\right),\left(f_{1}\right)$ and $\left(f_{2}\right)$ hold. If $\lambda=\lambda_{1}$, then $u=0$ is an isolated critical point of $I$. 
Proof By contradiction, assume that $\left\|u_{n}\right\| \rightarrow 0, I^{\prime}\left(u_{n}\right)=0$ and $u_{n} \not \equiv 0$, then by the elliptic estimates we have

$$
\left\|u_{n}\right\|_{C(\Omega)} \rightarrow 0, \quad \text { as } n \rightarrow \infty,
$$

this together with $\left(f_{2}\right)$ and $u_{n} \not \equiv 0$ gives

$$
\begin{aligned}
a \lambda_{1} \int_{\Omega}\left|u_{n}\right|^{2} d x & \leq a \int_{\Omega}\left|\nabla u_{n}\right|^{2} d x \\
& =-b\left\|u_{n}\right\|^{4}+a \lambda_{1} \int_{\Omega}\left|u_{n}\right|^{2} d x+\int_{\Omega} g\left(x, u_{n}\right) u_{n} d x \\
& <a \lambda_{1} \int_{\Omega}\left|u_{n}\right|^{2} d x, \quad \text { for } n \text { large enough, }
\end{aligned}
$$

which is a contradiction. Then $u=0$ is an isolated critical point of the functional $I$.

Assume that $\theta \in C^{1}(\mathbb{R},[-\alpha, \alpha])$ is a non-decreasing mapping with

$$
\theta(u)= \begin{cases}u & \text { if }|u| \leq \alpha / 2 \\ \pm \alpha & \text { if } \pm u \geq \alpha\end{cases}
$$

where $\alpha$ is defined in $\left(f_{2}\right)$. We define a functional $\Phi_{\tau} \in C^{1}\left(H_{0}^{1}(\Omega), \mathbb{R}\right)$ by setting (see for example [6, Lemma 4.4])

$$
\Phi_{\tau}(u)=\frac{a}{2}\|u\|^{2}+\frac{b}{4}\|u\|^{4}-\frac{a \lambda_{1}}{2} \int_{\Omega}|u|^{2} d x-\int_{\Omega} G(x,(1-\tau) u+\tau \theta(u)) d x,
$$

where $G(x, u)=\int_{0}^{u} g(x, t) d t$, and $\tau \in[0,1]$. Moreover, for any $\tau \in[0,1]$, by $\left(f_{0}\right)$ we also know that $\Phi_{\tau}(u)$ satisfies (P.S) condition on any bounded domain in $H_{0}^{1}(\Omega)$.

Proof of Theorem 1.2 By Lemma 3.1, we know that $u=0$ is an isolated critical point of $I$.

(1) First, we want to prove that there is a ball $\bar{B}_{\varepsilon}(0)$ with $\varepsilon>0$ small enough such that

$$
K\left(\Phi_{\tau}\right) \cap \bar{B}_{\varepsilon}(0)=\{0\}, \quad \forall \tau \in[0,1] .
$$

By contradiction, we assume there exist sequences $\tau_{n} \in[0,1]$ and $u_{n} \in H_{0}^{1}(\Omega) \backslash\{0\}$ such that

$$
\Phi_{\tau_{n}}^{\prime}\left(u_{n}\right)=0, \quad u_{n} \rightarrow 0(n \rightarrow \infty) .
$$

By the elliptic estimates we have

$$
\left\|u_{n}\right\|_{C(\Omega)} \rightarrow 0, \quad \text { as } n \rightarrow \infty,
$$

which implies that, for $n$ big enough,

$$
u_{n} \in \bar{B}_{\varepsilon}(0), \quad \text { and } \quad\left\|u_{n}\right\|_{C(\Omega)} \leq \alpha / 2 \text {, }
$$


then $\theta\left(u_{n}\right)=u_{n}$ and $\Phi_{0}\left(u_{n}\right)=\Phi_{\tau_{n}}\left(u_{n}\right)$ by definition (3.1). Now, for $n$ big enough, we get

$$
I^{\prime}\left(u_{n}\right)=\Phi_{0}^{\prime}\left(u_{n}\right)=\Phi_{\tau_{n}}^{\prime}\left(u_{n}\right)=0
$$

which is a contradiction with the fact that $u=0$ is an isolated critical point of $I$ in Lemma 3.1. Then (3.2) is true.

(2) Next, we want to prove

$$
C_{*}\left(\Phi_{1}, 0\right)=\delta_{*, 0} \mathbb{F} \text {. }
$$

Indeed, from (3.1), we know $|\theta(u)| \leq \alpha$ for $u \in H_{0}^{1}(\Omega)$, then $\left(f_{2}\right)$ gives

$$
\begin{aligned}
\Phi_{1}(u) & =\frac{a}{2}\|u\|^{2}+\frac{b}{4}\|u\|^{4}-\frac{a \lambda_{1}}{2} \int_{\Omega}|u|^{2} d x-\int_{\Omega} G(x, \theta(u)) d x \\
& \geq-\int_{\Omega} G(x, \theta(u)) d x \\
& \geq 0
\end{aligned}
$$

which implies that 0 is a local minimizer of $\Phi_{1}$, so (3.3) is true.

(3) Now, by (3.2), (3.3) and the homotopy invariance of the critical groups in Proposition 2.2 , we have

$$
C_{*}(I, 0)=C_{*}\left(\Phi_{0}, 0\right)=C_{*}\left(\Phi_{1}, 0\right)=\delta_{*, 0} \mathbb{F} .
$$

The proof is completed.

\section{Proof of Theorem 1.3}

We introduce two truncated energy functionals by setting

$$
I_{ \pm}(u)=\frac{a}{2}\|u\|^{2}+\frac{b}{4}\|u\|^{4}-\int_{\Omega} F_{ \pm}(x, u) d x, \quad u \in H_{0}^{1}(\Omega)
$$

where $F_{ \pm}(x, u)=\int_{0}^{u} f_{ \pm}(x, s) d s$ and

$$
f_{ \pm}(x, t)= \begin{cases}f(x, t) & \pm t \geq 0 \\ 0 & \pm t<0\end{cases}
$$

Clearly $I_{ \pm} \in C^{1}\left(H_{0}^{1}(\Omega), \mathbb{R}\right)$. Obviously, a nonzero critical point $u_{ \pm}$of $I_{ \pm}$is a nontrivial nonnegative (non-positive) solution of problem (1.1). Indeed, for any $w \in H_{0}^{1}(\Omega)$ we have

$$
0=\left\langle I_{+}^{\prime}\left(u_{+}\right), w\right\rangle=a \int_{\Omega} \nabla u_{+} \nabla w d x+b\left\|u_{+}\right\|^{2} \int_{\Omega} \nabla u_{+} \nabla w d x-\int_{\Omega} f_{+}\left(x, u_{+}\right) w d x
$$

Choosing $w=u_{+}^{-}=\min \left\{u_{+}, 0\right\}$, we get $\left\|u_{+}^{-}\right\|=0$. Therefore, $u_{+} \geq 0$ for a.e. $x \in \Omega$. The case for $I_{-}$is similar.

Lemma 4.1 If $\left(f_{0}\right)$ and $\left(f_{3}\right)$ hold, then I and $I_{ \pm}$satisfy the (P.S) condition. 
Proof It suffices to show that $I$ and $I_{ \pm}$are coercive on $H_{0}^{1}(\Omega)$. The following method is similar to [7]. For the functional $I$, by contradiction, there is a sequence $\left\{u_{n}\right\} \subset H_{0}^{1}(\Omega)$ such that

$$
I\left(u_{n}\right) \leq C, \quad \text { as }\left\|u_{n}\right\| \rightarrow \infty .
$$

If we set $v_{n}=\frac{u_{n}}{\left\|u_{n}\right\|}$, then $\left\|v_{n}\right\|=1$ and there is a $v_{0} \in H_{0}^{1}(\Omega)$ such that

$$
\begin{cases}v_{n} \rightarrow v_{0}, & \text { weakly in } H_{0}^{1}(\Omega), \\ v_{n} \rightarrow v_{0}, & \text { strongly in } L^{4}(\Omega), \\ v_{n}(x) \rightarrow v_{0}(x), & \text { a.e. } x \in \Omega\end{cases}
$$

From the condition $\left(f_{3}\right)$, we get

$$
\int_{\Omega}\left(F(x, u)-\frac{b \mu_{1}}{4}|u|^{4}\right) d x \leq \beta \int_{\Omega} u^{2} d x+C, \quad \text { for any } u \in H_{0}^{1}(\Omega), x \in \Omega
$$

this together with (4.1) gives

$$
\frac{C}{\left\|u_{n}\right\|^{4}} \geq \frac{I\left(u_{n}\right)}{\left\|u_{n}\right\|^{4}} \geq \frac{a}{2}\left\|u_{n}\right\|^{-2}+\frac{b}{4}\left\|v_{n}\right\|^{4}-\frac{b \mu_{1}}{4} \int_{\Omega}\left|v_{n}\right|^{4} d x-\frac{C+C\left\|u_{n}\right\|^{2}}{\left\|u_{n}\right\|^{4}}
$$

then we obtain

$$
\limsup _{n \rightarrow \infty}\left\|v_{n}\right\|^{4} \leq \mu_{1} \int_{\Omega}\left|v_{0}\right|^{4} d x
$$

On the other hand, by the variational characterization of $\mu_{1}$ and the lower semicontinuity of the norm we get

$$
\mu_{1} \int_{\Omega}\left|v_{0}\right|^{4} d x \leq\left\|v_{0}\right\|^{4} \leq \liminf _{n \rightarrow \infty}\left\|v_{n}\right\|^{4}
$$

which implies that

$$
\lim _{n \rightarrow \infty}\left\|v_{n}\right\|^{4}=\left\|v_{0}\right\|^{4}
$$

and

$$
\left\|v_{0}\right\|^{4}=\mu_{1} \int_{\Omega}\left|v_{0}\right|^{4} d x
$$

Therefore

$$
v_{n} \rightarrow v_{0} \quad \text { strongly in } H_{0}^{1}(\Omega)
$$

Then we get $\left|v_{0}\right| \neq 0$ for a.e. $x \in \Omega$, and by (4.2) we also have

$$
\left|u_{n}(x)\right| \rightarrow+\infty, \quad \text { a.e. } x \in \Omega \text {. }
$$


Using $\beta<\frac{a \lambda_{1}}{2},(4.3)$ and (4.4) give

$$
\begin{aligned}
I\left(u_{n}\right) & \geq \frac{a}{2} \int_{\Omega}\left|\nabla u_{n}\right|^{2} d x-\int_{\Omega}\left(F\left(x, u_{n}\right)-\frac{b \mu_{1}}{4}\left|u_{n}\right|^{4}\right) d x \\
& \geq \frac{a \lambda_{1}}{2} \int_{\Omega}\left|u_{n}\right|^{2} d x-\beta \int_{\Omega}\left|u_{n}\right|^{2} d x-C \\
& \rightarrow+\infty, \quad \text { as } n \rightarrow \infty,
\end{aligned}
$$

this is a contradiction with (4.1).

The case of $I_{+}\left(I_{-}\right)$is similar.

Let $e_{1}>0$ be the eigenfunction associated with $\lambda_{1}$.

Lemma 4.2 If $\left(f_{1}\right)$ with $k \geq 2$ holds, then there exists $t>0$ such that $I_{ \pm}\left( \pm t e_{1}\right)<0$.

Proof By $\left(f_{0}\right)$ and $\left(f_{1}\right)$, for $\varepsilon>0$ with $\lambda_{k}-\varepsilon>\lambda_{1}$ there exists $2<v \leq 2^{*}$ such that

$$
F(x, u) \geq \frac{a}{2}\left(\lambda_{k}-\varepsilon\right) u^{2}-C|u|^{v}, \quad \forall u \in \mathbb{R}, x \in \Omega .
$$

Then we get

$$
\begin{aligned}
I_{+}\left(t e_{1}\right) \leq & \frac{a \lambda_{1}|t|^{2}}{2} \int_{\Omega}\left|e_{1}\right|^{2} d x+\frac{b|t|^{4}}{4}\left\|e_{1}\right\|^{4} \\
& -\frac{a\left(\lambda_{k}-\varepsilon\right) t^{2}}{2} \int_{\Omega}\left|e_{1}\right|^{2} d x+C|t|^{\nu} \int_{\Omega}\left|e_{1}\right|^{\nu} d x \\
\leq & -C|t|^{2}+C|t|^{4}+C|t|^{\nu} \\
< & 0, \quad \text { as } t>0 \text { small. }
\end{aligned}
$$

The other case is similar.

Proof of Theorem $1.3 I, I_{ \pm}$are coercive on $H_{0}^{1}(\Omega)$ and satisfy the (P.S) condition by Lemma 4.1. From Lemma 4.2, the functional $I$ has a positive critical point $u_{1}$ and a negative critical point $u_{2}$ such that

$$
C_{*}\left(I, u_{1}\right)=C_{*}\left(I, u_{2}\right)=\delta_{*, 0} \mathbb{F}
$$

Using the mountain pass lemma in [1], we know that equation has a solution $u_{3}$ such that (see [2])

$$
C_{1}\left(I, u_{3}\right) \neq 0 \text {. }
$$

Moreover, using $\left(f_{1}\right)$ with $k \geq 2$, Theorem 1.1 gives

$$
C_{*}(I, 0)=\delta_{*, k} \mathbb{F},
$$

which implies that $u_{3} \neq 0$. The proof is completed. 


\title{
5 Conclusions
}

There are many difficulties if we want to obtain critical groups estimates for the Kirchhoff type equation; for example, we are not sure if the generalized Morse splitting lemma can be used. Then in this paper, by using the basic properties that critical groups are invariant under homotopies preserving the isolatedness of critical points, we can compute critical groups at zero when we impose on $f$ the non-resonance and resonance conditions. Moreover, using these critical groups estimates our theorem can get more nontrivial solutions. The main results presented in this paper improve and generalize many results in $[4,19,25]$.

\author{
Acknowledgements \\ The authors thank Professor Jiabao Su for many valuable discussions and suggestions. \\ Funding \\ This paper is supported by the NSFC (11771302, 11601353, 1174013), the fund of Beijing Education Committee \\ (KM201710009012, 6943), the fund of North China University of Technology (XN018010, XN012).
}

Availability of data and materials
Not applicable.

Competing interests

The authors declare that they have no competing interests.

Authors' contributions

All authors contributed equally and significantly in writing this article. All authors read and approved the final manuscript.

\section{Author details}

${ }^{1}$ College of Sciences, North China University of Technology, Beijing, China. ${ }^{2}$ School of Mathematical Sciences, Capital Normal University, Beijing, China. ${ }^{3}$ Department of Mathematics, Beijing University of Chemical Technology, Beijing, China.

\section{Publisher's Note}

Springer Nature remains neutral with regard to jurisdictional claims in published maps and institutional affiliations.

Received: 4 September 2018 Accepted: 3 December 2018 Published online: 06 December 2018

References

1. Ambrosetti, A., Rabinowitz, P.H.: Dual variational methods in critical points theory and applications. J. Funct. Anal. 14, 349-381 (1973)

2. Chang, K.-C.: Infinite Dimensional Morse Theory and Multiple Solution Problems. Birkhäuser, Boston (1993)

3. Chang, K.-C., Ghoussoub, N.: The Conley index and the critical groups via an extension of Gromoll-Meyer theory. Topol. Methods Nonlinear Anal. 7, 77-93 (1996)

4. Cheng, B., Wu, X.: Existence results of positive solutions of Kirchhoff problems. Nonlinear Anal. 71, 4883-4892 (2009)

5. Fiscella, A., Pucci, P.: P-Fractional Kirchhoff equations involving critical nonlinearities. Nonlinear Anal., Real World Appl. 35, 350-378 (2017)

6. Iannizzotto, A., Liu, S., Perera, K., Squassina, M.: Existence results for fractional $p$-Laplacian problems via Morse theory. Adv. Calc. Var. 9, 101-125 (2016)

7. Jiu, Q., Su, J.: Existence and multiplicity results for Dirichlet problems with p-Laplacian. J. Math. Anal. Appl. 281, 587-601 (2003)

8. Kirchhoff, G.: Mechanik. Teubner, Leipzig (1883)

9. Li, S.-J., Willem, M.: Applications of local linking to critical point theory. J. Math. Anal. Appl. 189, 6-32 (1995)

10. Lions, J.L.: On some questions in boundary value problems of mathematical physics. In: Contemporary Developments in Continuum Mechanics and Partial Differential Equations. Proc. Internat. Sympos., Inst. Mat. Univ. Fed. Rio de Janeiro, Rio de Janeiro. North-Holland Math. Stud., vol. 30, pp. 284-346. North-Holland, Amsterdam (1978)

11. Liu, J.-Q:: A Morse index for a saddle point. Syst. Sci. Math. Sci. 2, 32-39 (1989)

12. Liu, J.-Q., Su, J.: Remarks on multiple nontrivial solutions for quasi-linear resonant problems. J. Math. Anal. Appl. 258, 209-222 (2001)

13. Mao, A., Zhang, Z.: Sign-changing and multiple solutions of Kirchhoff type problems without the P.S. condition. Nonlinear Anal. 70, 1275-1287 (2009)

14. Mawhin, J., Willem, M.: Critical Point Theory and Hamiltonian Systems. Springer, Berlin (1989)

15. Perera, K., Zhang, Z.: Nontrivial solutions of Kirchhoff-type problems via the Yang index. J. Differ. Equ. 221, 246-255 (2006)

16. Su, J.: Semilinear elliptic boundary value problems with double resonance between two consecutive eigenvalues. Nonlinear Anal. 48, 881-895 (2002)

17. Su, J.: Existence and multiplicity results for classes of elliptic resonant problems. J. Math. Anal. Appl. 273, 565-579 (2002) 
18. Sun, J., Liu, S.: Nontrivial solutions of Kirchhoff type problems. Appl. Math. Lett. 25, 500-504 (2012)

19. Sun, J., Tang, C.: Existence and multiplicity of solutions for Kirchhoff type equations. Nonlinear Anal. 74, 1212-1222 (2011)

20. Sun, M.: Multiplicity of solutions for a class of the quasilinear elliptic equations at resonance. J. Math. Anal. Appl. 386 661-668 (2012)

21. Sun, M., Zhang, M., Su, J.: Critical groups at zero and multiple solutions for a quasilinear elliptic equation. J. Math. Anal. Appl. 428, 696-712 (2015)

22. Sun, Y.: Indirect boundary integral equation method for the Cauchy problem of the Laplace equation. J. Sci. Comput. 71, 469-498 (2017)

23. Xiang, M., Zhang, B., Qiu, H.: Existence of solutions for a critical fractional Kirchhoff type problem in $\mathbb{R}^{N}$. Sci. China Math. 60, 1647-1660 (2017)

24. Yang, Y., Zhang, J.: Nontrivial solutions of a class of nonlocal problems via local linking theory. Appl. Math. Lett. 23, 377-380 (2010)

25. Zhang, B., Molica Bisci, G., Xiang, M.: Multiplicity results for nonlocal fractional $p$-Kirchhoff equations via Morse theory. Topol. Methods Nonlinear Anal. 49, 445-461 (2017)

26. Zhang, Z., Perera, K.: Sign changing solutions of Kirchhoff type problems via invariant sets of descent flow. J. Math. Anal. Appl. 317, 456-463 (2006)

\section{Submit your manuscript to a SpringerOpen ${ }^{\circ}$} journal and benefit from:

- Convenient online submission

- Rigorous peer review

- Open access: articles freely available online

- High visibility within the field

- Retaining the copyright to your article

Submit your next manuscript at $\gg$ springeropen.com 